\title{
A New Hyperfine Operator In The Dirac Equation
}

\author{
H. Pilkuhn \\ Institut für Theoretische Teilchenphysik \\ Universität Karlsruhe \\ Postfach 6980, D-76128 Karlsruhe
}

\begin{abstract}
A recent eight-component differential equation for the relativistic two-body problem in QED is reduced to four components for a discussion of its antihermitian part of the hyperfine operator. This part proves essential for the mixing of $j=f-\frac{1}{2}$ and $j=f+\frac{1}{2}$ states at fixed total angular momentum $f$. The 4-component version is extended to anomalous magnetic moments. An application to helium-like Rydberg states is formulated. CPT-invariance is demonstrated and discussed in connection with positronium.
\end{abstract} 849510

\section{Introduction}

A recent "double-Dirac" equation (Pilkuhn 1992, Pilkuhn and Stäudner 1993, Malvetti and Pilkuhn 1994) describes bound and scattering states of two relativistic Dirac particles by 8 components instead of 16 . Its hyperfine operator has been investigated for states with $f=\ell \pm 1$ and $f=0$ ( $f$ is the total angular momentum) and for particle $\mathrm{g}$-factors $g_{1}=g_{2}=2$, as in positronium and muonium. In this paper, the complete hyperfine operator is discussed also for states with $f=\ell>0$, and the equation is extended to arbitrary $\mathrm{g}$-factors of both particles. The equation is now applicable to atomic hydrogen, to antiprotonic atoms, and to Rydberg states of helium-like ions, where the inner electron endows the core with a g-factor of the order of -8000 . The new antihermitian part of the hyperfine operator is essential for the $2 p_{1 / 2}-2 p_{3 / 2}$ hyperfine mixing of $f=1$-states.

The equation refers to the $\mathrm{cm}$ system, $\boldsymbol{p}_{1}=\boldsymbol{p}_{2}=\boldsymbol{p}=-i \nabla(\hbar=c=$ $\left.1, \boldsymbol{r}=\boldsymbol{r}_{1}-\boldsymbol{r}_{2}\right)$, at total energy $E=E_{1}+E_{2}=m_{1}+m_{2}+E_{b}$, where $E_{b}$ is negative for bound states. It treats both particles on equal footing but has nevertheless asymmetric spin operators, which arise from an asymmetric elimination of components. The equation resembles a recoil-corrected Dirac equation,

$$
H \psi=\epsilon \psi, \quad H=V(r)+\mu \beta+\boldsymbol{\alpha}_{1}\left(\boldsymbol{p}+e \boldsymbol{A}_{h f}\right),
$$

where $\boldsymbol{A}_{h f}$ is the vector potential due to the nuclear magnetic moment, $\boldsymbol{A}_{h f}=\operatorname{rot} \mu_{2} / r, \mu_{2}=e \boldsymbol{\sigma}_{2} g_{2} / 2 \mathrm{~m}$. The nonrelativistic reduced mass is replaced by its relativistic counterpart,

$$
\mu=m_{1} m_{2} / E
$$


and the $\epsilon$ in (1.1) is a "reduced energy",

$$
\epsilon=\left(E^{2}-m_{1}^{2}-m_{2}^{2}\right) / 2 E, \quad \epsilon^{2}-\mu^{2}=k^{2} .
$$

Even in the incomplete equation (1.1), $\mu$ and $\epsilon$ are symmetric in particles 1 and 2, and $\boldsymbol{\alpha}_{1} \boldsymbol{p}$ accounts for the kinetic energies of both particles (the latter property is related to $\left.\left(\boldsymbol{\alpha}_{1} \boldsymbol{p}\right)^{2}=p^{2}=p_{1}^{2}=p_{2}^{2}\right)$.

To formulate the double-Dirac equation, one must rewrite $\boldsymbol{\alpha}_{1}$ as $\gamma_{5} \boldsymbol{\sigma}_{1}$, at least in connection with the new hyperfine operator (a complete set of matrices is constructed from $\gamma_{5}, \beta, \boldsymbol{\sigma}_{1}$ and $\left.\boldsymbol{\sigma}_{2}\right)$. For $g_{1}=g_{2}=2$, the equation reads (Malvetti and Pilkuhn 1994)

$$
\begin{gathered}
{\left[\pi^{0}-\mu \beta-\gamma_{5}\left(\boldsymbol{\sigma}_{1} \boldsymbol{p}+p_{h f}\right)\right] \psi=0} \\
\pi^{0}=\epsilon-V, \quad p_{h f}=p_{h}+p_{a}, \\
p_{h}=-\left(\boldsymbol{\sigma}_{1} \times \boldsymbol{\sigma}_{2}\right) \boldsymbol{r} V^{\prime} / 2 E r, \quad p_{\boldsymbol{a}}=i \boldsymbol{\sigma}_{2} \boldsymbol{r} V^{\prime} / 2 E r .
\end{gathered}
$$

The operator $\gamma_{5} p_{h}$ is identical with the hyperfine operator of (1.1), except that $g_{2} / 2 m_{2}$ is replaced by $1 / E$, which implies a recoil correction in addition to $g_{2}=2$. The antihermitian part $p_{a}$ of the "hyperfine momentum" $p_{h f}$ is new: its necessity for the the states with $\ell=f>0$ is demonstrated in the next section. A common treatment of all 4 hyperfine states is presented in section 3 .

A single particle $i$ with anomalous magnetic moment $\kappa_{i}$ in external electric and magnetic fields satisfies an "anomalous Dirac equation", in which the combination

$$
g_{i}=2\left(1+\kappa_{i}\right)
$$

appears in connection with a magnetic field, while a different combination appears in connection with an electric field. The correct combinations for the two-body problem with arbitrary masses are known from the nonrelativistic reduction of Breit operators; they are summarised in eq. (5.2) below. They have not yet been derived for the double-Dirac equation, but one can introduce free parameters into that equation and adjust these to the Breit operators. The parametrisation requires some knowledge of the corresponding problem for a spinless particle 2 , which is briefly recapitulated in section 4. For this "Klein-Dirac" equation, a straightforward derivation is available and agrees with the Breit operators. The 8-component double-Dirac equation with arbitrary $g_{1}$ and $g_{2}$ is simple only for $\ell=f \pm 1$ and for $f=0$, where $p_{a}$ is negligible:

$$
\begin{gathered}
{\left[\tilde{\pi}^{0}-\tilde{\mu}-\gamma_{5}\left(\boldsymbol{\sigma}_{1} \boldsymbol{p}+\tilde{p}_{h f}\right)\right] \psi=0} \\
\tilde{p}_{h}=-g_{1} g_{2}\left(\boldsymbol{\sigma}_{1} \times \boldsymbol{\sigma}_{2}\right) \hat{\boldsymbol{r}} / 8 E c, \quad \tilde{\pi}^{0} \approx \epsilon / c-c V
\end{gathered}
$$


The precise definitions of $\tilde{\pi}^{0}, \tilde{\mu}$ and $c$ are given later. They imply

$$
\tilde{\pi}^{02}-\tilde{\mu}^{2}=\pi^{02}-\mu^{2}=k^{2}-2 \epsilon V+V^{2}
$$

and guarantee that the new spin couplings do not affect the spinless operators. Atomic hydrogen has $c \approx g_{1} / 2=1.00116$.

\section{The hyperfine singlet-triplet mixing}

Starting from the 16-component Dirac-Breit equation for the four spin-quartets $\psi_{g g}, \psi_{f f}, \psi_{g f}$ and $\psi_{f g}(g=$ large component, $f=$ small component) and eliminating the combinations $\psi_{g g}-\psi_{f f}, \psi_{g f}-\psi_{f g}$, one obtains asymmetric equations with denominators $m=m_{1}+m_{2}$ and $\delta m=m_{1}-m_{2}$. In an explicit singlet-triplet basis, these are cancelled against corresponding factors in the numerators. A shift of variable, $r^{*}=r+\alpha / 2 E$, transforms $\left(E-V^{*}\right)^{2}$ into $E(E-2 V)$ and cancels many Breit operators. The factor $E$ is divided off; it is still visible in the denominators of $\mu$ and $\epsilon$. The details (Malvetti and Pilkuhn 1994) are omitted here; the final result is (1.4), including the antihermitian operator $p_{a}$ in $p_{h f}$. It is investigated below. In numerical calculations, $V^{\prime}$ in (1.6) may be replaced by the more precise expression $\left(V^{*}\right)^{\prime}=-V /(r+\alpha / E)$.

Antihermitian operators are avoidable but can be very useful. For the ordinary Dirac equation, they arise in the chiral basis $\psi_{R}=\psi_{g}+\psi_{f}, \psi_{L}=$ $\psi_{g}-\psi_{f}$ by elimination of $\psi_{L}$. Equation (1.4) is also of this type, but with $p_{h f}$ added to $\boldsymbol{\sigma}_{1} \boldsymbol{p}$ :

$$
\begin{gathered}
\left(\pi^{0}+\boldsymbol{p} \boldsymbol{\sigma}_{1}+p_{h f}\right)\left(\pi^{0}-\boldsymbol{p} \boldsymbol{\sigma}_{1}-p_{h f}\right) \psi_{R}=\mu^{2} \psi_{R} \\
\left(\pi^{02}-\mu^{2}-p^{2}+i \sigma_{1}, V^{\prime}-\left\{\boldsymbol{p} \boldsymbol{\sigma}_{1}, p_{h f}\right\}-p_{h f}^{2}\right) \psi_{R}=0, \quad \sigma_{1 r}=\boldsymbol{\sigma}_{1} \hat{\boldsymbol{r}}
\end{gathered}
$$

Irrespective of $p_{h f}$, this equation contains the antihermitian $i \sigma_{1 r} V^{\prime}$. For $p_{h f}=0, V^{\prime}=\alpha / r^{2}$, all $r^{-2}$-operators can be collected in one operator $\tilde{L}^{2} / r^{2}$,

$$
\tilde{L}^{2}\left(p_{h f}=0\right)=L^{2}-\alpha^{2}-i \alpha \sigma_{1 r} .
$$

Its eigenvalues can be written as $\ell^{\prime}\left(\ell^{\prime}+1\right)=\left(j+\frac{1}{2}\right)^{2}-\alpha^{2} \pm \gamma, \gamma=$ $\left[\left(j+\frac{1}{2}\right)^{2}-\alpha^{2}\right]^{1 / 2}$. The term $-\alpha^{2}$ in $\gamma$ arises as $(-i \alpha)^{2}=-\alpha^{2}$. Thus the shortest access to the eigenvalues of the point Dirac equation uses in fact the non-hermitian operator (2.3). For approximate calculations, Dirac's parity basis ( $\beta$ diagonal) is frequently more convenient. Elimination of the small components $\psi_{f}$ yields the following equation for $\psi_{g}$ :

$$
\pi_{-}^{0} \psi_{g}=\left(\boldsymbol{p} \boldsymbol{\sigma}_{1}+p_{h f}\right)\left(\pi_{+}^{0}\right)^{-1}\left(\boldsymbol{p} \boldsymbol{\sigma}_{1}+p_{h f}\right) \psi_{g}, \quad \pi_{ \pm}^{0}=\pi^{0} \pm \mu .
$$


Substituting $\psi_{g}=\sqrt{\pi_{+}^{0}} \psi_{g}^{\prime}$ and multiplying also by $\sqrt{\pi_{+}^{0}}$, one obtains a simpler equation without changing the hermiticity properties of the operators. To order $\alpha^{6}$, it reads

$$
\begin{aligned}
& \pi_{+}^{0} \pi_{-}^{0} \psi_{g}^{\prime}=\left\{p^{2}+\left[\boldsymbol{\sigma}_{1} \nabla, \frac{V^{\prime} \boldsymbol{\sigma}_{1} \boldsymbol{r}}{2 \pi_{+}^{0} r}\right]\right.+\left\{\boldsymbol{\sigma}_{1} \boldsymbol{p}, p_{h f}\right\}+i\left[p_{h f}, \frac{V^{\prime} \boldsymbol{\sigma}_{1} \boldsymbol{r}}{2 \pi_{+}^{0} r}\right] . \\
&\left.+p_{h f}^{2}+\left(\frac{V^{\prime}}{2 \pi_{+}^{0}}\right)^{2}\right\} \psi_{g}^{\prime}
\end{aligned} .
$$

Both in (2.2) and (2.5), the leading hyperfine operator is $\left\{\boldsymbol{\sigma}_{1} \boldsymbol{p}, p_{h f}\right\}$. Its hermitian part is

$$
\begin{gathered}
\left\{\boldsymbol{\sigma}_{1} \boldsymbol{p}, p_{h}\right\}=\boldsymbol{\sigma}_{2} \boldsymbol{L} V^{\prime} / E r+R \\
R=\left(\boldsymbol{\sigma}_{1} \boldsymbol{\sigma}_{2}\left[\nabla, \boldsymbol{r} V^{\prime} / r\right]-\boldsymbol{\sigma}_{2} \nabla\left(\boldsymbol{\sigma}_{1} \boldsymbol{r} V^{\prime} / \boldsymbol{r}\right)+\left(\boldsymbol{\sigma}_{1} \boldsymbol{r} V^{\prime} / r\right) \boldsymbol{\sigma}_{2} \nabla\right) / 2 E
\end{gathered}
$$

The term $\sigma_{2} L V^{\prime} / E r$ in (2.6) is the "other spin-orbit"-interaction, the rest $R$ contains the tensor and Fermi interactions. As these operators are symmetric in $\sigma_{1}$ and $\sigma_{2}$, we may exchange $\sigma_{1}$ with $\sigma_{2}$ in $R$. Adding now

$$
\left\{\boldsymbol{\sigma}_{1} \boldsymbol{p}, p_{a}\right\}=\left[\boldsymbol{\sigma}_{1} \nabla\left(\boldsymbol{\sigma}_{2} \boldsymbol{r} V^{\prime} / r\right)+\left(\boldsymbol{\sigma}_{2} \boldsymbol{r} V^{\prime} / r\right) \boldsymbol{\sigma}_{1} \nabla\right] / 2 E,
$$

one finds

$$
\left.\left\{\boldsymbol{\sigma}_{1} \boldsymbol{p}, p_{h f}\right\}=\left(\boldsymbol{\sigma}_{2} \boldsymbol{L} V^{\prime} / r\right)+\frac{1}{2} \boldsymbol{\sigma}_{1} \boldsymbol{\sigma}_{2}\left[\nabla, \boldsymbol{r} V^{\prime} / r\right]+\boldsymbol{\sigma}_{2 r} V^{\prime} \boldsymbol{\sigma}_{1} \nabla\right) / E
$$

Finally, one may for $V=-\alpha / r$ omit all hyperfine operators which contribute only to s-waves, and calculate the $\ell=0$ hyperfine shifts by analytic continuation in $\ell$ (except for an annihilation graph in positronium, which is omitted here). Writing $\boldsymbol{\sigma}_{1} \nabla=\sigma_{1 r}\left(\partial_{r}-\boldsymbol{L} \boldsymbol{\sigma}_{1} / r\right)$, one may neglect the operators $r^{-2} \partial_{r}$ and $\left[\nabla, \boldsymbol{r} V^{\prime} / r\right]=\nabla^{2} V$ in (2.9):

$$
\left\{\boldsymbol{p} \boldsymbol{\sigma}_{1}, p_{h f}\right\}=\left(-\sigma_{1 r} \sigma_{2 r} \boldsymbol{L} \boldsymbol{\sigma}_{1}+\boldsymbol{L} \boldsymbol{\sigma}_{2}\right) V^{\prime} / E r .
$$

To order $V^{\prime} / r$, the only other spin operator in (2.5) is the familiar spin-orbit interaction,

$$
\left[\boldsymbol{\sigma}_{1} \nabla, V^{\prime} \boldsymbol{\sigma}_{1} \boldsymbol{r}\right] / r(\epsilon+\mu)=\boldsymbol{L} \boldsymbol{\sigma}_{1} V^{\prime} / r(\epsilon+\mu)
$$

To this order, all spin operators of (2.5) are now proportional to $L$. For parity eigenstates, two of the four components of $\psi_{g}$ vanish. In this section, we only treat the the parity $(-1)^{f}$. The two relevant values of $L^{2}\left(p^{2}=p_{r}^{2}-L^{2} / r^{2}\right.$ in 2.5) are then degenerate, and the non-hermitian component of (2.10) becomes essential.

In the triplet singlet basis of the $\ell=f$-subspace, one has, with $F=$ $\sqrt{f(f+1)}$

$$
\chi_{n}=\left(\begin{array}{c}
\chi_{f, 1} \\
\chi_{f, 0}
\end{array}\right), \boldsymbol{L} \boldsymbol{\sigma}_{1}=\left(\begin{array}{cc}
-1 & F \\
F & 0
\end{array}\right), \boldsymbol{L} \boldsymbol{\sigma}_{2}=\left(\begin{array}{cc}
-1 & -F \\
-F & 0
\end{array}\right) .
$$


Note that $L \sigma_{1}+L \sigma_{2}=2 L S$ is diagonal. Moreover,

$$
\sigma_{1 r} \sigma_{2 r}=\left(\begin{array}{cc}
1 & 0 \\
0 & -1
\end{array}\right)
$$

To order $\alpha^{4}$, the difference between $\epsilon$ and $\mu$ is negligible in (2.11). A factor $-2 \epsilon F^{2}$ will be separated, such that the matrix to be diagonalised in (2.5) is $-F^{2} A V^{\prime} / \epsilon r$,

$$
-A=\left[\boldsymbol{L} \boldsymbol{\sigma}_{1}+2 \hat{\epsilon}\left(\boldsymbol{L} \boldsymbol{\sigma}_{2}-\sigma_{1 r} \sigma_{2 r} \boldsymbol{L} \boldsymbol{\sigma}_{1}\right)\right]=\frac{1}{2 F^{2}}\left(\begin{array}{cc}
-1 & F(1-4 \hat{\epsilon}) \\
F & 0
\end{array}\right),
$$

with $\hat{\epsilon}=\epsilon / E$. Its eigenvalues are

$$
-a_{\ell}^{( \pm)}=\left(1 \pm \sqrt{1+4 F^{2}(1-4 \hat{\epsilon})}\right) / 4 F^{2}
$$

They agree with the eigenvalues of the Breit operators, which will be discussed in section 5 . Without the antihermitian $\left\{\boldsymbol{\sigma}_{1} \boldsymbol{p}, p_{a}\right\}$, each $F$ in the matrix $(2.14)$ would be multiplied by $(1-2 \hat{\epsilon})$, which would produce a factor $(1-2 \hat{\epsilon})^{2}$ instead of $1-4 \hat{\epsilon}$ under the square root in $(2.5)$. This would contradict the positronium spectra, where $m_{1}=m_{2} \approx E / 2$ leads to $\hat{\epsilon}=1 / 4$,

$$
a_{\ell}^{(+)}=-1 / 2 F^{2}, \quad a_{\ell}^{(-)}=0 .
$$

These simple values reflect the conservation of the total spin $S=\left(\sigma_{1}+\sigma_{2}\right) / 2$ for equal masses. One sees that the new hyperfine operator contributes only to second-order recoil effects. For states with $\ell=f>0$, however, it does contribute in first-order relativity. With the approximation $\pi_{+}^{0}=\epsilon-V+\mu \approx 2 \epsilon$ in the $\alpha^{6}$-effects, $(2.5)$ becomes

$$
\begin{gathered}
\left(k^{2}+2 \epsilon \alpha / r+r^{-1} \partial_{r}^{2} r-r^{-2}\left(F^{2}-\alpha^{2}\right)+2 F^{2} A \alpha / r^{2}[(r(\epsilon+\mu)+\alpha]\right. \\
\left.-p_{h f}^{2}-i\left[p_{h f}, \alpha \sigma_{1 r} / 2 \epsilon r^{2}\right]-\alpha^{2} / 4 \mu^{2} r^{4}\right) \psi_{g}^{\prime}=0 .
\end{gathered}
$$

The substitution $\psi_{g}^{\prime}=r^{-1} u$ transforms $r^{-1} \partial_{r}^{2} r$ into $\partial_{r}^{2}$. With $A$ diagonal, one can define a new distance $r^{\prime}$ and expand to first order in $\alpha / \mu$

$$
r=r^{\prime}-a \alpha / 2 \mu, \quad\left(F^{2}-\alpha^{2}\right) / r^{2}=\left(F^{2}-\alpha^{2}\right) / r^{\prime 2}+F^{2} a / \mu r^{3},
$$

which removes the $r^{-3}$-operators to this order. The oparator $2 \epsilon \alpha / r$ becomes

$$
2 \epsilon \alpha / r=2 \epsilon \alpha / r^{\prime}+\alpha^{2} a / r^{\prime 2} .
$$

In this way one obtains from (2.17)

$$
\left(k^{2}+2 \epsilon \alpha / r+\partial_{r}^{2}-\tilde{L}^{2} / r^{2}\right) u=0, \quad \tilde{L}^{2}=L^{2}-\alpha^{2}(1+a) .
$$

This equation may be solved exactly. 


\section{The four-component chiral form}

For $\ell=f$, one can diagonalise the spin operator $A V^{\prime} / r$ (2.14) simultaneously with $L^{2} / r^{2}$. For states of parity $(-1)^{f+1}, L^{2}$ has the two different eigenvalues $(f+1)(f+2)$ and $f(f-1)$, and $L^{2} / r^{2}$ cannot be diagonalized together with $A V^{\prime} / r$ any longer. One may then calculate the hyperfine splitting perturbatively from the original form (1.4) (Pilkuhn and Stäudner 1994), or one can transform $r^{-3}$ into $r^{-2}$ and then diagonalise the complete matrix $M / r^{2}$. Here the latter method is chosen. The chiral basis is now used because it preserves the exact Dirac limit. But as the chiral basis contains both parities, one must consider here the full $4 \times 4$-matrices.

With $p_{h f}^{2} \approx 0$ in (2.2) and $\left\{\boldsymbol{p} \boldsymbol{\sigma}_{1}, p_{h f}\right\}$ from (2.10), one gets

$$
\begin{gathered}
\left(k^{2}+2 \epsilon \alpha / r+\alpha^{2} / r^{2}+\partial_{r}^{2}-\left(L^{2}-i \alpha \sigma_{1 r}\right) / r^{2}+M_{h f} \alpha / E r^{3}\right) \psi_{R}=0 \\
M_{h f}=\sigma_{1 r} \sigma_{2 r} \boldsymbol{L} \boldsymbol{\sigma}_{1}-\boldsymbol{L} \boldsymbol{\sigma}_{2}
\end{gathered}
$$

The operator $M_{h f} \alpha / E r^{3}$ is removed by the substitution

$$
\psi_{R} \rightarrow\left(1+\alpha M_{h f} \partial_{r} / 2 E L^{2}\right) \psi
$$

When the substitution bracket is pulled to the left in (3.1), it produces only two important commutators. The first one is $\left[M_{h f} \partial_{r} / L^{2}, L^{2} / r^{2}\right] \alpha / 2 E$, which cancels $M_{h f} \alpha / E r^{3}$. The other is the commutator with $2 \epsilon \alpha / r$, which produces $M_{h f} \alpha^{2} \epsilon / E L^{2} r^{2}$ (operators $r^{-2} \partial_{r}$ are neglected). The sum of all $r^{-2}$-operators has the form $\tilde{L}^{2}$,

$$
\tilde{L}^{2}=L^{2}-\alpha^{2}-i \alpha \sigma_{1 r}-\alpha \hat{\epsilon} M_{h f} / L^{2}, \quad \hat{\epsilon}=\epsilon / E .
$$

In addition to the pair $\chi_{n}$ (2.12) of spin-angular functions, we now need a second pair which is initially taken in a basis $p$ in which $L^{2}, \boldsymbol{L} \boldsymbol{\sigma}_{1}$ and $\boldsymbol{L} \boldsymbol{\sigma}_{2}$ are all diagonal. Omitting for brevity the zeros of diagonal matrices, one has

$$
\chi_{p}=\left(\begin{array}{c}
\chi_{f+1} \\
\chi_{f-1}
\end{array}\right), L^{2}=\left(\begin{array}{c}
(f+1)(f+2) \\
(f-1) f
\end{array}\right), \boldsymbol{L} \boldsymbol{\sigma}_{1}=\boldsymbol{L} \boldsymbol{\sigma}_{2}=\left(\begin{array}{c}
-f-2 \\
f-1
\end{array}\right) .
$$

The matrices $\boldsymbol{L} \boldsymbol{\sigma}_{i} / L^{2}$ are obtained simply by dividing with the relevant eigenvalues of $L^{2}$. In (2.14), this eigenvalue is $F^{2}$, and in (3.5), it is also convenient to separate a factor $1 / F^{2}$ :

$$
\left(\boldsymbol{L} \boldsymbol{\sigma}_{i} / L^{2}\right)_{p}=\frac{1}{F^{2}}\left(\begin{array}{c}
-f \\
f+1
\end{array}\right) .
$$

In this way, the complete $4 \times 4$-matrices $\boldsymbol{L} \boldsymbol{\sigma}_{i} / L^{2}$ acquire a common factor $1 / F^{2}$. In the following, however, the pair $\chi_{p}$ will be replaced by the "tensorial 
basis" $\chi_{t}$, in which the tensor operator $\sigma_{1 r} \sigma_{2 r}$ is diagonal (Malvetti and Pilkuhn 1994): $\chi_{p}=U \chi_{t}$,

$$
\chi_{t}=\left(\begin{array}{l}
\chi_{f+} \\
\chi_{f-}
\end{array}\right), U=\frac{1}{\sqrt{2 f+1}}\left(\begin{array}{cc}
\sqrt{f+1} & \sqrt{f} \\
-\sqrt{f} & \sqrt{f+1}
\end{array}\right), \sigma_{1 r} \sigma_{2 r}=\left(\begin{array}{cc}
-1 & 0 \\
0 & 1
\end{array}\right) .
$$

In the full quartet $\chi, \sigma_{1 r}$ and $\sigma_{2 r}$ are now antidiagonal:

$$
\chi=\left(\begin{array}{c}
\chi_{t} \\
\chi_{n}
\end{array}\right), \sigma_{1 r}=-\left(\begin{array}{cccc}
0 & 0 & 0 & 1 \\
0 & 0 & 1 & 0 \\
0 & 1 & 0 & 0 \\
1 & 0 & 0 & 0
\end{array}\right), \sigma_{2} r=\left(\begin{array}{cccc}
0 & 0 & 0 & 1 \\
0 & 0 & -1 & 0 \\
0 & -1 & 0 & 0 \\
1 & 0 & 0 & 0
\end{array}\right) .
$$

The $2 \times 2$ matrices (3.5) are not diagonal in the tensor basis:

$$
\begin{gathered}
L^{2}=\left(\begin{array}{cccc}
F^{2}+2 & 2 F & 0 & 0 \\
2 F & F^{2} & 0 & 0 \\
0 & 0 & F^{2} & 0 \\
0 & 0 & 0 & F^{2}
\end{array}\right) \\
\frac{\boldsymbol{L} \boldsymbol{\sigma}_{2}}{L^{2}}=\frac{1}{F^{2}}\left(\begin{array}{cccc}
0 & -F & 0 & 0 \\
-F & 1 & 0 & 0 \\
0 & 0 & -1 & -F \\
0 & 0 & -F & 0
\end{array}\right), \\
\sigma_{1 r} \sigma_{2 r} \frac{\boldsymbol{L} \boldsymbol{\sigma}_{1}}{L^{2}}=\frac{-1}{F^{2}}\left(\begin{array}{cccc}
0 & -F & 0 & 0 \\
F & -1 & 0 & 0 \\
0 & 0 & 1 & -F \\
0 & 0 & F & 0
\end{array}\right) .
\end{gathered}
$$

The difference of the two matrices (3.10) and (3.11) enters $M_{h f}$; this difference has nonzero elements $\pm 2 / F$ in only two positions. In the diagonalization of $\tilde{L}^{2}$, one may omit a multiple of the unit matrix. In view of the form (3.9) of $L^{2}$, we omit $F^{2}$ and diagonalize the matrix

$$
\tilde{L}^{2}-F^{2}+\alpha^{2}=\left(\begin{array}{cccc}
2 & 2 F-2 \alpha^{2} \hat{\epsilon} / F & 0 & i \alpha \\
2 F & 0 & i \alpha & 0 \\
0 & i \alpha & 0 & -2 \alpha^{2} \hat{\epsilon} / F \\
i \alpha & 0 & 0 & 0
\end{array}\right) .
$$

This will be postponed to section 5 , where the matrix is generalised for the accomodation of g-factors. 


\section{Dirac equation with arbitrary g-factor for particle 1 , but with a spinless nucleus.}

For a spinless nucleus, the hyperfine interaction vanishes, and particle 1 sees only an electric field. For this special case, the "anomalous Dirac" equation becomes

$$
\left[\pi^{0}-\mu \beta-\boldsymbol{\alpha}_{1}(\boldsymbol{p}-i a \beta \nabla V / 2)\right] \psi=0,
$$

with $a=\kappa_{1} / m_{1}$ in the static limit, $\kappa_{1}$ being the anomalous magnetic moment (1.7). For electrons and muons, $\kappa_{1}=\alpha / 2 \pi=0.00116$ is a radiative correction which could also be added perturbatively. We shall need (4.1) with a suitable generalisation of $a$, wich produces a relativistic two-body equation with one spinless constituent, as a help for the more general case of the next section.

The new operator $i \boldsymbol{\alpha}_{1} a \beta \nabla V / 2$ of (4.1) is removed by the substitution (Malvetti and Pilkuhn 1994)

$$
\psi=e^{\beta \eta / 2} \tilde{\psi}, \quad \eta=\eta_{0}-a V,
$$

and by the simultaneous transformation of $\pi^{0}$ and $\mu$ :

$$
\tilde{\pi}^{0}=\pi^{0} \cosh \eta+\mu \sinh \eta, \quad \tilde{\mu}=\mu \cosh \eta+\pi^{0} \sinh \eta .
$$

This leaves $\pi^{02}-\mu^{2}$ invariant as mentioned in (1.10), but it makes $\tilde{\mu}$ a function of $r$. The free parameter $\eta_{0}$ is chosen such that $\tilde{\mu}$ has no terms linear in $V=-\alpha / r$ :

$$
\begin{gathered}
e^{\eta_{0}}=[1+a(\mu-\epsilon)]^{1 / 2}[1+a(\mu+\epsilon)]^{-1 / 2}, \\
\tilde{\mu}_{0}=\frac{1}{2}(\mu+\epsilon) e^{\eta_{0}}+\frac{1}{2}(\mu-\epsilon) e^{-\eta_{0}}=\left(\mu-a k^{2}\right) / c_{10}:=w / c_{10}, \\
c_{10}^{2}=[1+a(\mu-\epsilon)][1+a(\mu+\epsilon)]=1+2 a \mu-a^{2} k^{2}, \\
w=\left(\epsilon^{2}-c_{10}^{2} k^{2}\right)^{1 / 2}=\left(\mu^{2}-\left(c_{10}^{2}-1\right) k^{2}\right)^{1 / 2} .
\end{gathered}
$$

Including terms quadratic in $V$, one has

$$
\tilde{\mu}(r)=w / c_{10}+a c_{10} V^{2}\left(1+a^{2} k^{2} / 2 c_{10}^{2}\right) .
$$

To order $\alpha^{4}$, the $V^{2}$-term in $\tilde{\mu}$ is required only in (1.10); its commutator with $\boldsymbol{\alpha}_{1} \boldsymbol{p}$ is negligible. In this approximation, the chiral component $\psi_{R}$ of $\tilde{\psi}$ satisfies an equation closely related to $(2.2)$ for $p_{h f}=0$ :

$$
\left(\pi^{02}-\mu^{2}-p^{2}+i c_{10} \sigma_{1 r} V^{\prime}\right) \psi_{R}=0 .
$$

It has two different static limits: Dirac for $c_{10}=1$ and Klein-Gordon for $c_{10}=0$. All recoil is included in the spin-free operators $\pi^{0}, \mu, \boldsymbol{p}$. For $c_{10}=0$, the equation is known as the Todorov equation (todorov 1971). The precise inclusion of recoil for all $E,-\infty<E<\infty$ requires (Pilkuhn 1984)

$$
c_{10}^{2}=1+2 \hat{\kappa}_{1}-\hat{m}_{1}^{2}, \quad \hat{m}_{i}=m_{i} / E, \quad \hat{\kappa}_{1}=\hat{m}_{2} \kappa_{1} .
$$


In the following, the asymptotic normalised single-particle energies $\hat{E}_{i}$ (in the region $V=0$ ) will also be needed:

$$
\hat{m}_{i}^{2}=\hat{E}_{i}^{2}-k^{2} / E_{i}^{2}, \quad \hat{E}_{1}+\hat{E}_{2}=1 .
$$

It is presently not known whether the precise value of $\hat{\kappa}_{1}$ is $\hat{m}_{2} \kappa_{1}$ or $\hat{E}_{2} \kappa_{1}$. One should also remember that a field theory based on the anomalous Dirac equation would not be renormalisable. For antiprotonic atoms such as antiprotonic helium, $\kappa_{1}$ must be included in (4.10) as it does not follow from radiative corrections with a local proton field.

\section{Adaption to two g-factors.}

The nonrelativistic reduction of Breit operators is normally combined with the Schrödinger equation, but the Todorov equation is equally suited (Pilkuhn 1982); spin-free operators such as $\nabla^{4} / 8 m_{1}^{3}$ are then absent:

$$
\left(k^{2}+\partial_{r}^{2}+2 \epsilon \alpha / r-\left(L^{2}-\alpha^{2}\right) / r^{2}-2 \mu V_{s}\right) u=0 .
$$

The spin potential $V_{s}$ including g-factors (Barmo et al 1981) is

$$
\begin{gathered}
2 \mu V_{s}=\left(\boldsymbol{L} \boldsymbol{S}_{1} c_{10}^{2}+\boldsymbol{L} \boldsymbol{S}_{2} c_{20}^{2}+2 g_{1} g_{2} S_{12}\right) V^{\prime} / r, \\
S_{12}=3 S_{1 r} S_{2 r}-\boldsymbol{S}_{1} \boldsymbol{S}_{2},
\end{gathered}
$$

where $c_{20}^{2}$ is analogous to $c_{10}^{2}$. Operators $\delta(r)$ are again omitted because $r^{-3}$ will be transformed into $r^{-2}$ by the method of section 2 . In this way, one arrives again at eq. (2.2), but this time with the following four eigenvalues $a_{\ell}$ of the spin operator $A$ :

$$
\begin{gathered}
a_{\ell}^{( \pm)}=-\left\{2 c_{0} \pm\left[4 c_{0}^{2}+4 F^{2}\left(c_{10}^{2}-c_{20}^{2}\right)^{2}\right]^{1 / 2}\right\} / 4 F^{2} \\
a_{f-1}=-g_{1} g_{2} \hat{\epsilon} / 2(2 f+1)-c_{0} / f, a_{f+1}=-g_{1} g_{2} \hat{\epsilon} / 2(2 f+1)+c_{0} /(f+1), \\
2 c_{0}=c_{10}^{2}+c_{20}^{2}-g_{1} g_{2} \hat{\epsilon} / 2=1+2 \hat{\kappa}_{1}+2 \hat{\kappa}_{2}+\hat{\epsilon}\left(2-g_{1} g_{2} / 2\right)
\end{gathered}
$$

For $g_{1}=g_{2}=2,2 c_{0}=1,(5.4)$ agrees with (2.15) as expected, and the $a_{f \pm 1}$ agree also with expectations (see below). To adapt (3.12) to (5.4) and (5.5) for general g-factors, one needs three free parameters, which can be introduced as follows into the matrix:

$$
\tilde{L}^{2}-F^{2}+\alpha^{2}=\left(\begin{array}{cccc}
2 & 2 F-2 \alpha^{2} \hat{\epsilon}_{t} / F & 0 & i c \alpha \\
2 F & 0 & i c \alpha & 0 \\
0 & i c \alpha & 0 & -2 \alpha^{2} \hat{\epsilon}_{n} / F \\
i c \alpha & 0 & 0 & 0
\end{array}\right),
$$

with $\hat{\epsilon}_{t} \neq \hat{\epsilon}_{n} \neq \hat{\epsilon}$. The antidiagonal part of (5.7) is $-i c \alpha \sigma_{1 r}$ as in (4.9), but with $c_{10}$ replaced by a third free parameter $c$. The four eigenvalues $\lambda$ of 
(5.7) follow from $\left|\tilde{L}^{2}-F^{2}+\alpha^{2}-\lambda\right|=0$. Neglecting a small term $\alpha^{6} \hat{\epsilon}_{t} \hat{\epsilon}_{n}$, this leads to

$$
\lambda^{2}\left[\lambda^{2}-2 \lambda-4\left(F^{2}-\alpha^{2} \hat{\epsilon}_{t}\right)+2 c^{2} \alpha^{2}\right]-2 c^{2} \alpha^{2} \lambda-4 c^{2} \alpha^{4} \hat{\epsilon}_{n}+c^{4} \alpha^{4}=0 .
$$

Eq.(5.9) may be cast into a form adapted to the static limit $\hat{\epsilon} \rightarrow 0$ :

$$
\begin{gathered}
f_{-} f_{+}-4\left(\hat{\epsilon}_{n}+\hat{\epsilon}_{t}\right) c^{2} \alpha^{4}-16 \hat{\epsilon}_{t}^{2} F^{2} /(2 f+1)^{2}=0, \\
f_{-}=\lambda^{2}+c^{2} \alpha^{2}+2 \lambda f+4 \alpha^{2} \hat{\epsilon}_{t} f /(2 f+1), \\
f_{+}=\lambda^{2}+c^{2} \alpha^{2}-2 \lambda(f+1)+4 \alpha^{2} \hat{\epsilon}_{t}(f+1) /(2 f+1) .
\end{gathered}
$$

The Dirac structure emerges if one sets $\lambda=\lambda_{-}-f$ in $f_{-}$and $\lambda=\lambda_{+}+f+1$ in $f_{+}$. Two of the four $\lambda$-values are of the order of $\alpha^{2}$. They are obtained by neglecting $\lambda^{4}$ and $\lambda^{3}$ in (5.8):

$$
\lambda=-\alpha^{2}\left(c^{2} \pm\left[c^{4}+4 F^{2}\left(c^{2}-4 \hat{\epsilon}_{n}\right)\right]^{1 / 2}\right) / 4 F^{2} .
$$

Comparison between (5.12) and (5.4) shows

$$
c^{2}=2 c_{0}, \quad c^{2}\left(c^{2}-4 \hat{\epsilon}_{n}\right)=\left(c_{10}^{2}-c_{20}^{2}\right)^{2} .
$$

These expressions suggest the definition of shifted constants $c_{i}^{2}$,

$$
c_{i}^{2}=c_{i 0}^{2}-g_{1} g_{2} \hat{\epsilon} / 4, \quad c_{1}^{1}+c_{2}^{2}=c^{2}=2 c_{0},
$$

such that $c^{4}$ can be rewritten as $\left(c_{1}^{2}+c_{2}^{2}\right)^{2}=\left(c_{1}^{2}-c_{2}^{2}\right)^{2}+4 c_{1}^{2} c_{2}^{2}$. This leads to

$$
\hat{\epsilon}_{n}^{2}=c_{1}^{2} c_{2}^{2} / c^{2}
$$

And for $\ell=f \pm 1$, (5.5) leads to

$$
\hat{\epsilon}_{t}=g_{1} g_{2} \hat{\epsilon} / 4 \text {. }
$$

Here however, the total of $-2 \alpha^{2} \hat{\epsilon}_{t} / F$ can be distributed freely among the two "2F-positions" in (5.7), since possible terms $\alpha^{4} \hat{\epsilon}_{t}^{2}$ are neglected in (5.5).

For $g_{1}=g_{2}=2$, one expects of course $c^{2}=1$ and $\hat{\epsilon}_{t}=\hat{\epsilon}_{n}=\hat{\epsilon}$. These expectations are fulfilled for $c^{2}=2 c_{0}$ (5.6) and for $\hat{\epsilon}_{t}$ (5.16) but not for $\hat{\epsilon}_{n}$ (5.15). With

$$
c_{1}^{2}\left(\kappa_{1}=0\right)=\hat{E}_{2}=\frac{1}{2}+\left(m_{2}^{2}-m_{1}^{2}\right) / 2 E^{2},
$$

the combination entering $\hat{\epsilon}_{n}$ is

$$
c_{1}^{2} c_{2}^{2}=\hat{E}_{1} \hat{E}_{2}=\hat{\epsilon}-k^{2} / E^{2} .
$$

The discrepancy $\hat{\epsilon}-\hat{\epsilon}_{n}=k^{2} / E^{2}$ contributes to the $\ell=f$ hyperfine splitting only at the order $\alpha^{6}$, but it is disturbing because its origin is unknown. One can now translate (5.7) into operators for use in the chiral equation (3.1),

$$
\left(k^{2}+2 \epsilon \alpha / r+\alpha^{2} / r^{2}-p^{2}+i c \alpha \sigma_{1 r} / r^{2}+M_{h f} \alpha / E r^{3}\right) \psi_{R}=0,
$$




$$
\begin{gathered}
M_{h f}=\left(\sigma_{1 r} \sigma_{2 r}-1\right) \boldsymbol{L} \boldsymbol{\sigma} g_{1} g_{2} / 8-\left(\sigma_{1 r} \sigma_{2 r}+1\right) \boldsymbol{L} \Delta \boldsymbol{\sigma} \epsilon_{n} / 2 \epsilon, \\
\boldsymbol{\sigma}=\boldsymbol{\sigma}_{1}+\boldsymbol{\sigma}_{2}, \quad \Delta \boldsymbol{\sigma}=\boldsymbol{\sigma}_{1}-\boldsymbol{\sigma}_{2}
\end{gathered}
$$

For $g_{2}=0$ and $c_{2}=0$, this equation reduces to (4.9), where particle 2 is assumed spinless. A more convenient form for comparable masses is

$$
\begin{gathered}
\left(k^{2}+2 \epsilon \alpha / r+\partial_{r}^{2}-\tilde{L}^{2} / r^{2}\right) u_{R}=0, \\
\tilde{L}^{2}=L^{2}-\alpha^{2}-i c \alpha \sigma_{1 r}+\alpha^{2}\left[\left(1-\sigma_{1 r} \sigma_{2 r}\right) \boldsymbol{L} \boldsymbol{\sigma} \hat{\epsilon}_{t}+\left(1+\sigma_{1 r} \sigma_{2 r}\right) \boldsymbol{L} \Delta \boldsymbol{\sigma} \hat{\epsilon}_{n}\right] / 2 L^{2}
\end{gathered}
$$

In general, it seems difficult to bring (5.20) into the factorising form (2.1), which is necessary for the original 8-component version. For the states with $\ell=f \pm 1$ and $f=0$, however, $p_{a}$ is negligible and the final result is (1.9).

\section{One-Electron atoms and helium-like Rydberg states.}

Our g-factor $g_{2}$ refers to a Zeeman operator $H_{Z e e}=Z e g_{2} S \boldsymbol{B} / 2 m_{2}$. The normal definition of a nuclear $g$-factor replaces $m_{2}$ by the proton mass $m_{2}$ and omits the nuclear charge $Z$. Consequently,

$$
g_{2}=g_{N} m_{2} / Z m_{p}
$$

The proton has $g_{2}=g_{N}=5.58, \mathrm{He}_{3}$ has $g_{2}=-3.185$ (Malvetti and Pilkuhn 1994). For ordinary electronic atoms, relativistic recoil effects are so small that the distinction between normal and anomalous nuclear magnetic moments is unnecessary. It is then useful to replace $2 \kappa_{2}$ by $g_{2}-2$ in $c_{2}^{2}$ (5.14):

$$
c_{2}^{2}=1-\hat{m}_{2}^{2}-2 \hat{m}_{1}+g_{2}\left(\hat{m}_{1}-g_{1} \hat{\epsilon} / 4\right) .
$$

The first three terms in (6.2) are negligible. In analogy with (5.17) one has $1-\hat{m}_{2}^{2}=2 \hat{E}_{1}-\hat{m}_{1}^{2}$,

$$
1-\hat{m}_{2}^{2}-2 \hat{m}_{1}=\hat{m}_{1}\left(2 E_{1} / m_{1}-2-\hat{m}_{1}\right) .
$$

With the nonrelativistic estimate $E_{1} \approx m_{1}\left(1-\alpha^{2} / 2 n^{2}\right)$, this reduces to $-\hat{m}_{1}\left(\hat{m}_{1}+\alpha^{2} / n^{2}\right) \approx 0$. For $c_{1}^{2}$, there are no further simplifications:

$$
c_{1}^{2}=c_{10}^{2}-g_{1} g_{2} \hat{\epsilon} / 4 \text {, }
$$

with $c_{10}^{2}$ from (4.10). But the sum $c^{2}=c_{1}^{2}+c_{2}^{2}$ can again be simplified:

$$
c^{2}=c_{10}^{2}+g_{2}\left(\hat{m}_{1}-\left(1+\kappa_{1}\right) \hat{\epsilon}\right) \text {. }
$$

Using now $\hat{\epsilon}=\hat{E}_{1}-\hat{m}_{1}^{2}$ and $\kappa_{1} \hat{\epsilon} \approx \kappa_{1} \hat{m}_{1}$, one obtains

$$
c^{2}=c_{10}^{2}+g_{2} \hat{m}_{1}\left(1-E_{1} / m_{1}+\hat{m}_{1}-\kappa_{1}\right) \text {. }
$$

The last bracket is again small, such that one has roughly $c^{2}=c_{10}^{2}$.

Consider now heliumlike Rydberg states. At sufficiently large orbital angular momentum $\ell$, the outer electron avoids the core, where the inner 
electron is firmly bound to the nucleus. For a spinless nucleus, the core has the magnetic moment $-e g_{1} / 4 m_{1}$ of its electron, with $g_{1}=2.0023$. Treating the core as a Dirac particle of charge $Z^{\prime}=\mathrm{Z}-1$ and g-factor $g_{2}$, the same magnetic moment appears in the form $Z^{\prime} e g_{2} / 4 m_{2}$. Consequently,

$$
g_{2}=-g_{1} m_{2} / m_{1} Z^{\prime} \text {. }
$$

This leads to

$$
\begin{gathered}
c_{1}^{2}=c_{10}^{2}+g_{1}^{2} \hat{m}_{2} \epsilon / 4 m_{1} Z^{\prime} \approx c_{10}^{2}+\hat{m}_{2} / Z^{\prime}, \\
c_{2}^{2} \approx-g_{1} \hat{m}_{2}\left[1-\left(1+\kappa_{1}\right) \epsilon / 2 m_{1}\right] / Z^{\prime} \approx-\hat{m}_{2} / Z^{\prime} .
\end{gathered}
$$

One may now also approximate $\hat{m}_{2}$ by $1, c_{1}^{2}=1+1 / Z^{\prime}, c_{2}^{2}=-1 / Z^{\prime}$,

$$
\hat{\epsilon}_{t} \approx-1 / Z^{\prime}, \quad \hat{\epsilon}_{n} \approx-\left(1+1 / Z^{\prime}\right) / Z^{\prime}
$$

The resulting spin structure is so large that a separation into fine and hyperfine structures is meaningfull only for large $Z^{\prime}$. These effects are already well known to order $Z^{\prime 3} \alpha^{4}$ from the nonrelativistic reduction of Breit operators, of course (Drake 1993). The new relativistic effects are of the order $Z^{\prime 5} \alpha^{6}$ and will be elaborated elsewhere.

\section{CPT, projectors and positronium.}

Eq.(1.4) has a symmetry which is either hidden or lost in other 8component equations: Multiplication by $E$ yields Malvetti and Pilkuhn 1994)

$$
\begin{gathered}
H_{s} \psi=e \psi, \quad e=E \epsilon=\frac{1}{2}\left(s-m_{1}^{2}-m_{2}^{2}\right), \quad s=E^{2}, \\
H_{s}=V_{\rho}+m_{1} m_{2} \beta+\boldsymbol{p}_{\rho} \boldsymbol{\alpha}_{1}-\gamma_{5}\left(\boldsymbol{\sigma}_{1} \times \boldsymbol{\sigma}_{2} \nabla_{\rho} V_{\rho}+\left[\boldsymbol{\sigma}_{2} \boldsymbol{p}_{\rho}, V_{\rho}\right]\right) / 2 s \\
\boldsymbol{\rho}=\boldsymbol{r} / E . \quad V_{\rho}=-\alpha / \rho .
\end{gathered}
$$

In this form (1.4) depends only on $s=E^{2}$ (g-factors are omitted in this section). Each bound state $s_{n}$ has two energy eigenvalues, $E_{n}^{+}=\sqrt{s_{n}}$ and $E_{n}^{-}=-\sqrt{s_{n}}$. For normal binary atoms with one electron and one nucleus, the state of energy $E_{n}^{-}$represents an antiatom of $\mathrm{cm}$ energy $-E_{n}^{-}=E_{n}^{+}$. For positronium and other CP-eigenstates, $E_{n}^{+}$and $E_{n}^{-}$belong to the same state.

The CPT-transformation for a single field operator $\Psi\left(x_{1}^{\mu}\right)=\Psi\left(t, \boldsymbol{r}_{1}\right)$ is

$$
\Psi^{\prime}\left(-x_{\mu}\right)=-\gamma_{5} \Psi\left(x_{\mu}\right)
$$

The 16-component wave function of the Dirac-Breit equation is constructed from matrix elements of two field operators at a common time, $t_{1}=t_{2}=t$. The corresponding CPT-transformation matrix is $\gamma_{15} \gamma_{25}$; it exchanges large components with small ones. As mentioned in section 2, (1.4) uses the 
eight components $\psi_{g g}+\psi_{f f}$ and $\psi_{g f}+\psi_{f g}$; these are not mixed with other components by CPT.

Although the symmetry looks trivial in the form $H_{s} \psi=e \psi$, it is well hidden in the from (1.4). At fixed $\rho=r / E$, it requires

$$
E \rightarrow-E, \quad r \rightarrow-r .
$$

The latter equation means a negative distance, $r=-\sqrt{r^{2}}$. Whereas the boundary conditions on $\psi(\boldsymbol{\rho})$ in (7.1) are at $\rho=0$ and $\rho=\infty$ as usual, those for $\psi(r)$ must be taken at $r=0, r=\infty$ for positive $E$ and at $r=0, r=\infty$ for negative $E$. Fortunately, there exists a general method of calculating energy eigenvalues which does not require explicit boundary conditions (Balslev and Combes1971, Reinhard 1976). It uses a Schrödinger equation, which in view of $(2.20)$ or (5.22) may be written as

$$
H \psi=k^{2} \psi, \quad H=\tilde{p}^{2}+2 \epsilon V, \quad \tilde{p}^{2}=p_{r}^{2}+\tilde{L}^{2} / r^{2} .
$$

The Hamiltonian of (7.6) is "dilatation analytic", which implies that its bound state spectrum is independent of the "dilatation" $\boldsymbol{r}=\boldsymbol{r}^{\prime} e^{i \Theta}$. Due to spherical symmetry, it will only be needed for complex distances,

$$
r=r^{\prime} e^{i \Theta} \text {, }
$$

leaving the angles real. We choose $\Theta=\pi$, which leads to negative distances, $r^{\prime}=-r$. This gives $V^{\prime}=-V$ in (7.6), which is compensated by a sign change of $E$ in $\epsilon V$. The operator $\tilde{L}^{2}$ is an even function of $E$, such that the equation is in fact invariant under (7.5). This symmetry should not be confused with the socalled substitution rule, which states that the wave functions for the $e^{+} p$ system are obtained from the Dirac equation for $e^{-} p$ at negative $\epsilon$, without changing the sign of $V$. The $e^{+} p$-system has no bound states, of course.

The original Dirac-Breit equation contains "positive-energy" projectors $\left(m_{e}=\right.$ electron mass)

$$
\lambda_{i+}=\frac{1}{2}\left[1+\left(m_{e} \beta_{i}+\boldsymbol{\alpha}_{i} \boldsymbol{p}_{i}\right) / H_{0 i}\right], \quad H_{0 i}=\sqrt{m_{e}^{2}+p_{i}^{2}} .
$$

These are necessary for binary atoms whenever a Breit operator $B$ is needed beyond first-order perturbation theory (Bethe and Salpeter 1957). They have been used in the derivation of (1.4) for the elimination of $B^{2}$, but they disappear from the result. One can redefine the $\lambda_{i+}$ as "equal-sign projectors", which refer to the signs of the single-particle energies relative to $E$. For negative $E$, one merely has to use the negative sign of the square root $H_{0 i}$. For the alternative formulation in the variable $\boldsymbol{\rho}=\boldsymbol{r} / E$, one may rewrite the second half of $\lambda_{i+}$ as

$$
\left(m_{e} \beta_{i}+\boldsymbol{\alpha}_{i} \boldsymbol{p}_{i}\right) / H_{i 0}=\left(E m_{e} \beta_{i}+\boldsymbol{\alpha}_{i} \boldsymbol{p}_{\rho i}\right) / \sqrt{m_{e}^{2} s+p_{\rho i}^{2}},
$$


and then always use the positive sign of the square root.

In atoms with many electrons, the electronic state has a higher symmetry when the nuclear degrees of freedom are ignored. For example, neglect of the hyperfine interaction leads to a conserved electronic angular momentum. Similarly, neglect of the nuclear recoil leads to a conserved electronic energy $E_{e}$. In this approximation, the atom-antiatom symmetry degenerates to a symmetry under $E_{e} \rightarrow-E_{e}, r_{i} \rightarrow-r_{i}, r_{i j} \rightarrow-r_{i j}$, i.e. to all distances negative (in the simplest case of a hydrogen atom with an infinitely heavy point nucleus, this symmetry manifests itself in the fact that $E_{e}$ is given by a square root). The necessity of projectors in many-electron atoms has been emphasized repeatedly (Sucher 1980), but it has been demonstrated in numerical applications only recently (Indelicato and Desclaux 1993). A consistent formulation should also here use the equal-sign projectors (7.9), with $s$ replaced by $s_{e}$ in the static limit. Even the nonrelativistic nuclear recoil can be reformulated to conform with CPT-invariance.

Hamiltonians of the type (1.1) are not fundamental, they are sometimes called "quasi-Hamiltonians" since they depend on $E$. The main $E$ dependence comes from the reduced mass $\mu=m_{1} m_{2} / E$; it disappears in the from (7.2) (this is in fact the original form, from which a factor $E$ has been divided off as mentioned at the beginning of section 2). Thus $H_{s}$ depends on $E$ only in the hyperfine interaction. The situation changes again with the reduction to four components. After multiplication by $s$, eq. (2.20) has the form

$$
\left(\pi_{\rho}^{02}-m_{1}^{2} m_{2}^{2}-p_{\rho}^{2}+\alpha^{2} a_{\ell} / \rho^{2}\right) u=0, \quad \pi_{\rho}^{0}=e-V_{\rho}
$$

This has the $e$-dependence of a Klein-Gordon equation, with a residual $e$ dependence in the dimensionless $a_{\ell}$. For equal masses and $\ell=f$, this dependence disappears according to (2.16). It seems thus possible that at least in some cases, (7.10) is a true Klein-Gordon equation with respect to its e-dependence. This would greatly simplify its orthogonality relations and Greens functions. It would also allow one to replace $s$ by a "proper time" derivative $i \partial_{\tau}: \pi_{\rho}^{0}=\frac{1}{2} i \partial_{\tau}-m_{1}^{2}-V_{\rho}$, and thus allow for a bilocal free field theory of positronium.

The author would like to thank P. Indelicato and B. Melic for helpful discussions.

\section{References}

Balslev E and Combes J M 1971, Comm.Math.Phys. 22, 280

Barmo S, Pilkuhn H and Schlaile HG 1981, Z.Phys. A 301, 283

Bethe H A and Salpeter E E 1957 Quantum mechanics of one- and two-electron atoms (Berlin: Springer) 
Drake G W F 1993 Adv. in atomic, mol. and opt. phys 31, 1 Indelicato P and Desclaux J P 1993 Physica Scripta T46, 110 Malvetti M and Pilkuhn H 1994, Phys.Rep. 248, 1.

Martinis M and Pilkuhn H 1982 J. Phys B 15, 1797

Pilkuhn H 1982 J. Phys B 174061

Pilkuhn H 1992 J. Phys. B 25, 299

Pilkuhn H and Stäudner F 1993, Phys.Lett.A 178, 156

Reinhardt W P 1976, Int.J.Quant.Chem. 10, 359

Sucher J 1980 Phys Rev A 22, 348

Todorov I T 1971 Phys Rev D 3, 2351 
